\title{
Neuronal activity-dependent myelin repair after stroke
}

Wanyi $\mathrm{Jia}^{1 *}$, Yasmine Kamen ${ }^{1 *}$, Helena Pivonkova ${ }^{1 *}$ and Ragnhildur T. Káradóttir ${ }^{1,2}$

1. Wellcome - Medical Research Council Cambridge Stem Cell Institute \& Department of Veterinary Medicine, University of Cambridge, Cambridge, United Kingdom

2. Department of Physiology, BioMedical Center, Faculty of Medicine, University of Iceland, Reykjavik, Iceland

* equal contribution

Correspondence:

Ragnhildur T. Káradóttir, $\mathrm{PhD}$

Wellcome - MRC Stem Cell Institute

University of Cambridge

Tennis Court Road

Cambridge

CB2 1QR

email: rk385@cam.ac.uk 


\begin{abstract}
Brain tissue undergoes substantial activity-dependent reorganisation after stroke due to neuronal plasticity, leading to partial functional recovery in patients. Concurrent myelin repair is crucial for proper neuronal network function and reorganisation. Myelin repair after stroke might occur as myelin plasticity or as remyelination through the recruitment and differentiation of oligodendrocyte precursor cells (OPCs), which become myelin-forming oligodendrocytes (OLs). These two processes might share a similar guiding mechanism, which is postulated to depend on neuronal activity and glutamate signaling to OPCs. However, with ageing, the ability of OPCs to differentiate into myelinating OLs decreases due to changes in their ion channel and neurotransmitter receptor expression profile, rendering them less sensitive to neuronal activity. Because of their unique ability to replace damaged OLs, OPCs represent a potential therapeutic target for myelin repair in the context of stroke.
\end{abstract}




\section{Introduction}

Stroke represents one of the leading causes of death and long-term disability worldwide [1]. Most cases originate from blockage of blood flow within cerebral arteries, which often occur in the territory of the middle cerebral artery, leading to the development of large lesions encompassing both cortical and subcortical grey and white matter [2]. Smaller lesions often resulting in subtle neurological manifestation occur mostly in subcortical structures, particularly in the white matter, and frequently progress to vascular dementia [3-5]. These lesions have been lately recognised to contribute to degenerative central nervous system (CNS) diseases, such as Alzheimer's Disease [6].

The CNS grey matter is composed of neuronal cell bodies and their processes, along with glial cell types which include astrocytes, oligodendrocytes (OLs), oligodendrocyte precursor cells (OPCs), and microglia. In the ischemic core, all cell types suffer complete lack of energy, and the majority die. In the penumbra around the ischemic core, where partial blood flow is preserved, cell death may be specific to more vulnerable cell types. As damage in this area is partial, neuronal plasticity allows neurons in the penumbra and the surrounding healthy brain region, or peri-infarct, to reorganise and rewire to achieve functional recovery [7]. Because CNS white matter consists of neuronal axons and the four glial cell types, white matter ischemia might lead to selective glial cell death followed by demyelination. OLs have been shown to be vulnerable to oxidative stress, trophic factor deprivation and glutamate toxicity, making them highly susceptible to ischemia [811]. White matter might thus be especially vulnerable to ischemia, as demonstrated by the high frequency of white matter abnormalities in aged patients suffering from severe cognitive dysfunction, sensorimotor impairment, pain and emotional disorders [12].

Patients often undergo remarkable neurological improvements after stroke, which are enhanced by physical activity and rehabilitation [13]. The cellular and molecular correlates of functional recovery can be assigned to activity-dependent neuronal plasticity including axonal sprouting, dendritic morphology changes and alterations in synaptic connectivity [14]. Neuronal precursors stemming from the neurogenic niche in the subventricular zone (SVZ) might migrate to the site of injury to replace damaged neurons, although to a limited extent [15]. However, neuronal plasticity and circuit reorganization may not be sufficient for functional recovery, given the importance of myelin in neuronal circuit formation and function [16]. Thus, myelin regeneration alongside 
neuronal repair and circuit reorganization might significantly improve the outcome of stroke patients. Myelin repair could occur via myelin plasticity or remyelination. Myelin plasticity, through de novo myelination of previously unmyelinated axons by newly formed OLs and changes in the structure of already-myelinated axons, might play an important role in the tissue repair of the penumbral regions, where some neuronal structures are preserved and reorganised [17]. Repair in these areas might contribute to functional improvement of neurological outcome in stroke patients. Remyelination of previously myelinated axons by OPC recruitment from the surrounding tissue and differentiation into new myelin-forming OLs could occur after ischemia in the white matter or after selective OL loss in the penumbra of grey matter ischemia.

In this review, we focus on myelin plasticity and remyelination as potential therapeutic targets after stroke. These two processes might significantly contribute to proper nervous tissue repair and neurological improvement in stroke patients. We propose that myelin plasticity and remyelination share a common underlying mechanism, which could be targeted to enhance functional recovery. OLs are the only cell type in the adult brain that can be replaced by their precursors, OPCs, which persist in all brain regions throughout life $[18,19]$. This unique ability makes OPCs a potent and promising therapeutic target. Moreover, we address the mechanisms leading to limited myelin plasticity and remyelination in aged patients, where stroke and its therapy become more relevant because of their higher incidences.

\section{Neuronal Plasticity after Stroke}

Surviving neurons in the penumbra are capable of partial functional recovery, which potentially compensates for the lost functions due to neuronal death in the ischemic core [20]. Experimental stroke models have shown synaptic and distal dendritic growth [21], extensive dendritic spine turnover [20] and pruning [22], along with axonal remodeling [23] and synaptogenesis [24,25]. In many cases, increased neuronal activity can enhance neuronal grey matter repair. Neuronal stimulation has been shown to induce axonal remodeling in the corticospinal tract in humans and rodents, leading to motor map reorganisation and possible functional recovery [23,26,27]. Axonal sprouting and outgrowth can be promoted by neuronal stimulation in both the corticospinal tract and the motor cortex $[15,28,29]$. Cortical axonal remodeling and rewiring can be facilitated through the use of repetitive transcranial magnetic stimulation, which modifies the excitability of 
the motor cortex over treatment periods [30]. In large strokes where a large part of the hemisphere is lost, asynchronous therapy targeting the contralesional hemisphere can restore motor control through corticospinal tract rewiring, leading to motor recovery [31]. Peri-infarct axonal sprouting, which initiates within the first week after stroke incidence [32], continues for several months after injury with task-specific rehabilitation [33]. In addition, stroke-induced neurogenesis can also contribute to self-repair. Neurogenesis in the SVZ is enhanced by increased neuronal firing, as demonstrated through the stimulation of neuronal activity in murine stroke models, allowing neural stem progenitor cells to migrate to the injured region [34].

\section{Myelin Plasticity Supports Neuronal Function}

Given myelin's role as a potential modulator of neuronal networks [35,36], myelin plasticity might be critical in supporting rewiring after stroke. Myelin plasticity consists of de novo myelination of previously unmyelinated axons and changes to existing myelin sheaths, including internode number and length, myelin thickness and geometry of the nodal area in mature CNS tissue [17]. Continuous de novo myelination occurs in the healthy adult in both white and grey matter, where adult OPCs actively divide and differentiate to form myelinating OLs [37-40]. Sensory enrichment, motor learning and direct neuronal stimulation enhance this process [35,38,41,42], while neuronal deprivation through social isolation impairs myelination [43]. Myelin plasticity in adulthood recapitulates development, during which neuronal activity is important for proper myelin formation. Social isolation studies in both juvenile primates and mice showed decreased myelination $[44,45]$. Similarly, blocking auditory activity decreases myelin sheath thickness [46]. Given the evidence showing that new motor skill learning is dependent on white matter structural changes and de novo myelination $[35,47]$, functional recovery might depend on myelin plasticity.

Indeed, an increasing number of studies point to the importance of myelin and the existence of myelin plasticity alongside neuronal plasticity. In chronic stroke patients, hemispheric myelin asymmetry, which is computed by the ratio of contralesional to ipsilesional myelin through myelin water fraction, showed that increased asymmetry between the hemispheres is a significant predictor of impairment and functional loss [48,49]. Unlike previously believed, persistent myelin loss has also been evidenced in contralesional hemispheres of chronic ischemic stroke patients [50,51]. These observations may be attributed to overall inactivity during crucial brain 
reorganisation [52], which may lead to myelin loss and reduced synaptic connectivity. In subjects with one immobilised arm without any neurological deficit, immobilisation leads to reduced white matter integrity and cortical thinning in the contralateral motor output pathway. Forced use of the non-immobilised arm leads to increases in both white matter and cortical thickness in the contralateral hemisphere [53]. Similar mechanisms occur after stroke, where simultaneous axonal and myelin remodeling are shown in the motor cortex contralesional to stroke [54]. In fact, enhancing myelination after neonatal hypoxia partially rescues synaptogenesis and increases motor functional recovery [55]. Since myelin plasticity can occur in parallel with neuronal rewiring, which is often followed by motor recovery [56], it is reasonable to believe that myelin plasticity might contribute to functional recovery after stroke. Thus, promoting both neuronal rewiring and myelin plasticity could provide greater rehabilitation.

\section{Remyelination Following Stroke}

While myelin plasticity might contribute to circuit rewiring, remyelination could be necessary in the penumbra or during white matter stroke to salvage OL-depleted tissues [57]. Remyelination is the myelination of previously myelinated axons, which have lost their sheaths due to injury. This process is mostly studied in toxin-induced demyelinating models, where OL death leads to demyelinated lesions. OPCs are recruited to these sites and proliferate and differentiate into mature myelinating OLs [58]. A number of different cues are known to regulate remyelination, including cytokines, growth factors and neuronal activity [58]. Recent evidence indicates that remyelination occurs after stroke. In the murine stroke model, administration of nicotinamide, whose metabolite $\mathrm{NAD}^{+}$promotes OPC differentiation [59], leads to a gain in function and increased fractional anisotropy in the surrounding white matter fibers of the internal capsule [60]. Brain-derived neurotrophic factor (BDNF) treatment in rats with induced subcortical ischemia improves tract connectivity and increases OPCs proliferation [61]. Moreover, blockage of Nogo Receptor 1 signaling increases OPC differentiation in the peri-infarct and rescues remyelination [62].

\section{Neuronal Activity: a Common Mechanism?}

Several lines of evidence suggest that remyelination and myelin plasticity might represent two aspects of the same process, myelination in the adult. In both cases, OPCs differentiate into mature myelinating OLs $[35,63]$. In addition, the underlying mechanism may be similar, as both processes 
could be driven by neuronal activity. OPCs monitor the firing pattern of neurons as they express voltage-gated ion channels and glutamate receptors and receive synaptic inputs from neurons [10,11,64-66]. Overlapping populations of OPCs and postsynaptic neurons show synchronised spontaneous activity, indicating that OPCs can sense activity patterns from circuits [67].

An increasing number of studies indicate that neuronal activity regulates remyelination. In particular, glutamate signaling is crucial for remyelination, as blocking vesicular release, $\alpha$-amino3-hydroxy-5-methyl-4-isoxazolepropionic acid/kainate receptors (AMPA/KARs) or N-methyl-Daspartate receptors (NMDARs) prevents remyelination in ethidium bromide-induced white matter lesions $[68,69]$. Neuronal activity may also regulate myelin plasticity. Direct stimulation of neuronal circuits leads to OPC differentiation and de novo myelination in adult mice [41,42]. In addition, motor learning, which is driven by changes in neuronal activity [70], requires de novo myelination [17,35]. Moreover, social isolation in adult mice decreases myelination, presumably due to a decrease in neuronal activity following reduced sensory inputs [43].

Given that OPCs express glutamate receptors and form synapses with axons in adulthood and after injury $[64,69,71,72]$, we postulate that neuronal activity modulates both remyelination and myelin plasticity. Specifically, glutamate signaling through axon-OPC synapses might be an important input. Understanding this common mechanism might therefore allow the identification of therapeutic targets to promote myelination in adults, since both remyelination and myelin plasticity might be important for repair and functional recovery following stroke.

\section{Heterogeneity in OPCs}

As neuronal signaling to OPCs might regulate remyelination and myelin plasticity, understanding the functional properties of OPCs becomes crucial. Unlike previously believed, recent evidence suggests that OPCs are heterogeneous. For instance, several groups report differential gene expression patterns and response to growth factors with age [73-75]. White and grey matter OPCs respond differently to cytokines [76] and express different ion channels [75,77]. These channels, which are essential for OPCs to sense neuronal activity, are also differentially expressed in OPCs in an age- and region-dependent manner. Notably, when OPCs first appear, they lack all ion channels, but then gradually acquire voltage-gated potassium $\left(\mathrm{K}_{\mathrm{V}}\right)$ channels, voltage-gated sodium 
$(\mathrm{Nav})$ channels, AMPA/KARs and NMDARs at different rates and differentially between and within regions [75].

The proliferation and differentiation potential of OPCs can be linked to region, age, transcriptome and ion channel and neurotransmitter receptor expression, suggesting that OPCs exist in different functional states. Several OPC states were recently identified. Embryonic-like,'naïve' OPCs lack voltage-gated ion channels and glutamate receptors and are presumably incapable of sensing neuronal activity. OPCs then acquire $\mathrm{K}_{\mathrm{V}}$ channels and AMPA/KARs, before transitioning into a 'highly proliferative' state, which is characterised by large Nav membrane densities. OPCs 'primed' for differentiation have high $\mathrm{Na}$ and NMDAR membrane densities, indicative of high sensitivity to neuronal activity and glutamate signaling. Finally, 'quiescent' OPCs lack NMDARs, but have high AMPA/KAR membrane densities. Importantly, while the majority of OPCs in a certain region or at a particular age are in a particular state, a small proportion of OPCs are in other states. This supports the notion that OPCs heterogeneity rather indicates different functional states (Figure 1A) [75]. Moreover, OPC states correlate with age. In young adults, the majority of OPCs are in a proliferative or primed state. However, in one-year-old mice, primed OPCs have disappeared, and only $1 \%$ of OPCs are proliferating. These changes are concomitant with an increase in quiescent OPCs numbers [75].

These functional states might have important implications for myelination in adults. In particular, if both remyelination and myelin plasticity are dependent on neuronal activity, the capacity of OPCs to sense neuronal activity, which is dependent on their ion channel expression profile, becomes a crucial factor in understanding the molecular mechanism underlying remyelination and myelin plasticity. For instance, remyelination failure is often due to a block in differentiation [78], which has been linked to ageing [79]. Taking into consideration OPC states, this failure in differentiation might be due to the declining proportion of primed OPCs and the increasing number of quiescent OPCs in older mice [75]. OPCs present in human chronic multiple sclerosis lesions have been described as quiescent, as they neither proliferate nor differentiate [80]. Thus, characterising OPC states in the context of demyelinated lesions might provide a better understanding of myelin repair mechanisms. 
In contrast to remyelination, myelin plasticity has mostly been examined in young adult mice $[35,41,43]$, when the relative abundance of primed OPCs suggests that the capacity for myelin plasticity is optimal. Little is known about myelin plasticity in older mice, so it would be important to examine whether, like remyelination, myelin plasticity is hindered in old age, when the majority of OPCs become quiescent. One intriguing study shows that sensory stimulation promotes de novo myelination in the somatosensory cortex in one-year-old mice [38]. Unlike in many other brain areas, myelination continues in the upper layers of the somatosensory cortex in mice beyond a year and half [37-39], similar to comparative timelines in humans [17]. But by two years, myelin loss is observed in the somatosensory cortex, presumably due to the lack of OPCs differentiation, indicating that de novo myelination declines with age [37]. It is surprising how long myelination in the somatosensory cortex continues, given the loss of primed OPCs and the increasing proportion of quiescent OPCs in most brain areas in one-year-old mice. A possible explanation is the large number of SVZ OPCs that remain primed for differentiation beyond that age [75]. Perhaps this population is maintained for up to two years and is the source of newly formed cortical OLs. Another possibility is that somatosensory cortex OPCs differ from other cortical OPCs and remain primed into old age. However, this is currently unknown. Alternatively, sensory stimulation could lead to the release of growth factors like BDNF or neuregulin and reactivate NMDAR expression in OPCs [68]. This promotes a shift towards the primed state and thus allows for activity-dependent myelin plasticity. A better understanding of OPC states and the extent of state transition might unveal the dynamics and underlying mechanism of remyelination and myelin plasticity. This in turn could provide targets to enhance myelination in adults, particularly during ageing.

\section{Future Perspectives}

The capacity of OPCs to differentiate into mature myelinating OLs in response to neuronal activity makes them an important target to enhance myelin plasticity and remyelination. Because myelin plasticity and remyelination are important processes in nervous tissue repair after stroke, therapeutic strategies should target both neurons and OPCs to better promote functional recovery.

\section{Acknowledgements}


This work was supported by the European Research Council (ERC) under the European Union's Horizon 2020 research and innovation programme (grant agreement No 771411; R.T.K.); a Wellcome Studentship (102160/Z/13/Z; Y.K.), a Wellcome Pathfinder Award (204488/Z/16/Z; R.T.K. \& H.P); The Gates Foundation, a Gates Scholarship (W.J.), The UK MS Society, a Cambridge Myelin Repair Centre grant (50; R.T.K, H.P.); The Fonds de recherche du QuébecSanté, a scholarship (Y.K.); The Cambridge Commonwealth European \& International Trust, a scholarship (Y.K.); and the Lister Institute, a Research Prize (R.T.K.).

\section{References:}

[1] M. Katan, A. Luft, Global Burden of Stroke, Semin. Neurol. (2018). doi:10.1055/s-00381649503.

[2] R. Ornello, D. Degan, C. Tiseo, C. Di Carmine, L. Perciballi, F. Pistoia, A. Carolei, S. Sacco, Distribution and Temporal Trends From 1993 to 2015 of Ischemic Stroke Subtypes, Stroke. (2018). doi:10.1161/STROKEAHA.117.020031.

[3] S.E. Vermeer, W.T. Longstreth, P.J. Koudstaal, Silent brain infarcts: a systematic review, Lancet Neurol. (2007). doi:10.1016/S1474-4422(07)70170-9.

[4] J.B. Pinkston, N. Alekseeva, E. González Toledo, Stroke and dementia, Neurol. Res. (2009). doi:10.1179/016164109X12445505689643.

[5] A. Joutel, H. Chabriat, Pathogenesis of white matter changes in cerebral small vessel diseases: beyond vessel-intrinsic mechanisms, Clin. Sci. (2017). doi:10.1042/CS20160380.

[6] M.L. Alosco, M.A. Sugarman, L.M. Besser, Y. Tripodis, B. Martin, J.N. Palmisano, N.W. Kowall, R. Au, J. Mez, C. DeCarli, T.D. Stein, A.C. McKee, R.J. Killiany, R.A. Stern, A Clinicopathological Investigation of White Matter Hyperintensities and Alzheimer's Disease Neuropathology, J. Alzheimer's Dis. (2018). doi:10.3233/JAD-180017.

[7] U. Dirnagl, C. Iadecola, M.A. Moskowitz, Pathobiology of ischaemic stroke, Trends Neurosci. (1999). doi:10.1016/S0166-2236(99)01401-0.

[8] D.M. Holtzman, N.L. Luo, C.A. Chricton, K.L. Arvin, B.H. Han, J. Tam, S.A. Back, S. Xanthoudakis, Selective Vulnerability of Late Oligodendrocyte Progenitors to HypoxiaIschemia, J. Neurosci. (2018). doi:10.1523/jneurosci.22-02-00455.2002.

[9] D. Dewar, S.M. Underhill, M.P. Goldberg, Oligodendrocytes and ischemic brain injury, J. Cereb. Blood Flow Metab. (2003). doi:10.1097/01.WCB.0000053472.41007.F9. 
[10] R. Káradóttir, P. Cavelier, L.H. Bergersen, D. Attwell, NMDA receptors are expressed in oligodendrocytes and activated in ischaemia, Nature. (2005). doi:10.1038/nature04302.

[11] R. Káradóttir, N.B. Hamilton, Y. Bakiri, D. Attwell, Spiking and nonspiking classes of oligodendrocyte precursor glia in CNS white matter, Nat. Neurosci. (2008). doi:10.1038/nn2060.

[12] S. Paradiso, B.M. Anderson, L.L. Boles Ponto, D. Tranel, R.G. Robinson, Altered neural activity and emotions following right middle cerebral artery stroke, J. Stroke Cerebrovasc. Dis. (2011). doi:10.1016/j.jstrokecerebrovasdis.2009.11.005.

[13] L.I. Benowitz, S.T. Carmichael, Promoting axonal rewiring to improve outcome after stroke, Neurobiol. Dis. (2010). doi:10.1016/j.nbd.2009.11.009.

[14] S.T. Carmichael, B. Kathirvelu, C.A. Schweppe, E.H. Nie, Molecular, cellular and functional events in axonal sprouting after stroke, Exp. Neurol. (2017). doi:10.1016/j.expneurol.2016.02.007.

[15] K.J. Wu, S. Yu, J.Y. Lee, B. Hoffer, Y. Wang, Improving Neurorepair in Stroke Brain Through Endogenous Neurogenesis-Enhancing Drugs, Cell Transplant. (2017). doi:10.1177/0963689717721230.

[16] K.A. Nave, Myelination and support of axonal integrity by glia, Nature. (2010). doi:10.1038/nature09614.

[17] O. de Faria, E.A.C. Pama, K. Evans, A. Luzhynskaya, R.T. Káradóttir, Neuroglial interactions underpinning myelin plasticity, Dev. Neurobiol. (2018). doi:10.1002/dneu.22539.

[18] M.R.L. Dawson, A. Polito, J.M. Levine, R. Reynolds, NG2-expressing glial progenitor cells: An abundant and widespread population of cycling cells in the adult rat CNS, Mol. Cell. Neurosci. (2003). doi:10.1016/S1044-7431(03)00210-0.

[19] L.E. Rivers, K.M. Young, M. Rizzi, F. Jamen, K. Psachoulia, A. Wade, N. Kessaris, W.D. Richardson, PDGFRA/NG2 glia generate myelinating oligodendrocytes and piriform projection neurons in adult mice, Nat. Neurosci. (2008). doi:10.1038/nn.2220.

[20] C.E. Brown, P. Li, J.D. Boyd, K.R. Delaney, T.H. Murphy, Extensive Turnover of Dendritic Spines and Vascular Remodeling in Cortical Tissues Recovering from Stroke, J. Neurosci. (2007). doi:10.1523/JNEUROSCI.4295-06.2007.

[21] T.A. Jones, J.A. Kleim, W.T. Greenough, Synaptogenesis and dendritic growth in the cortex opposite unilateral sensorimotor cortex damage in adult rats: A quantitative electron microscopic examination, Brain Res. (1996). doi:10.1016/0006-8993(96)007925.

[22] T.A. Jones, T. Schallert, Overgrowth and pruning of dendrites in adult rats recovering from neocortical damage, Brain Res. (1992). doi:10.1016/0006-8993(92)90356-E. 
[23] N. Okabe, N. Himi, E. Maruyama-Nakamura, N. Hayashi, K. Narita, O. Miyamoto, Rehabilitative skilled forelimb training enhances axonal remodeling in the corticospinal pathway but not the brainstem-spinal pathways after photothrombotic stroke in the primary motor cortex, PLoS One. (2017). doi:10.1371/journal.pone.0187413.

[24] J. Chen, Z.G. Zhang, Y. Li, Y. Wang, L. Wang, H. Jiang, C. Zhang, M. Lu, M. Katakowski, C.S. Feldkamp, M. Chopp, Statins induce angiogenesis, neurogenesis, and synaptogenesis after stroke, Ann. Neurol. (2003). doi:10.1002/ana.10555.

[25] M.-K. Sun, J. Hongpaisan, T.J. Nelson, D.L. Alkon, Poststroke neuronal rescue and synaptogenesis mediated in vivo by protein kinase $\mathrm{C}$ in adult brains, Proc. Natl. Acad. Sci. (2008). doi:10.1073/pnas.0805952105.

[26] R.J. Nudo, G.W. Milliken, Reorganization of movement representations in primary motor cortex following focal ischemic infarcts in adult squirrel monkeys, J. Neurophysiol. (1996). doi:10.1152/jn.1996.75.5.2144.

[27] A.S. Wahl, U. Büchler, A. Brändli, B. Brattoli, S. Musall, H. Kasper, B. V. Ineichen, F. Helmchen, B. Ommer, M.E. Schwab, Optogenetically stimulating intact rat corticospinal tract post-stroke restores motor control through regionalized functional circuit formation, Nat. Commun. (2017). doi:10.1038/s41467-017-01090-6.

[28] M. Brus-Ramer, J.B. Carmel, S. Chakrabarty, J.H. Martin, Electrical Stimulation of Spared Corticospinal Axons Augments Connections with Ipsilateral Spinal Motor Circuits after Injury, J. Neurosci. (2007). doi:10.1523/JNEUROSCI.3489-07.2007.

[29] J.B. Carmel, J.H. Martin, Motor cortex electrical stimulation augments sprouting of the corticospinal tract and promotes recovery of motor function, Front. Integr. Neurosci. (2014). doi:10.3389/fnint.2014.00051.

[30] L. Lee, H.R. Siebner, J.B. Rowe, V. Rizzo, J.C. Rothwell, R.S.J. Frackowiak, K.J. Friston, Acute remapping within the motor system induced by low-frequency repetitive transcranial magnetic stimulation, J. Neurosci. 23 (2003) 5308-18. doi:23/12/5308 [pii].

[31] A.S. Wahl, W. Omlor, J.C. Rubio, J.L. Chen, H. Zheng, A. Schröter, M. Gullo, O. Weinmann, K. Kobayashi, F. Helmchen, B. Ommer, M.E. Schwab, Asynchronous therapy restores motor control by rewiring of the rat corticospinal tract after stroke, Science. (2014). doi:10.1126/science.1253050.

[32] S. Li, J.J. Overman, D. Katsman, S. V. Kozlov, C.J. Donnelly, J.L. Twiss, R.J. Giger, G. Coppola, D.H. Geschwind, S.T. Carmichael, An age-related sprouting transcriptome provides molecular control of axonal sprouting after stroke, Nat. Neurosci. (2010). doi:10.1038/nn.2674.

[33] K. Aminoltejari, H. Erb, C.E. Brown, T.H. Murphy, I.R. Winship, In Vivo VoltageSensitive Dye Imaging in Adult Mice Reveals That Somatosensory Maps Lost to Stroke Are Replaced over Weeks by New Structural and Functional Circuits with Prolonged Modes of Activation within Both the Peri-Infarct Zone and Distant Sites, J. Neurosci. 
(2009). doi:10.1523/jneurosci.4249-08.2009.

[34] M. Song, S.P. Yu, O. Mohamad, W. Cao, Z.Z. Wei, X. Gu, M.Q. Jiang, L. Wei, Optogenetic stimulation of glutamatergic neuronal activity in the striatum enhances neurogenesis in the subventricular zone of normal and stroke mice, Neurobiol. Dis. (2017). doi:10.1016/j.nbd.2016.11.005.

[35] W.D. Richardson, B. Emery, D. Ohayon, H. Li, J. Paes de Faria, K. Tohyama, I.A. McKenzie, Motor skill learning requires active central myelination, Science. (2014). doi:10.1126/science. 1254960 .

[36] S. Pajevic, P.J. Basser, R.D. Fields, Role of myelin plasticity in oscillations and synchrony of neuronal activity, Neuroscience. (2014). doi:10.1016/j.neuroscience.2013.11.007.

[37] R.A. Hill, A.M. Li, J. Grutzendler, Lifelong cortical myelin plasticity and age-related degeneration in the live mammalian brain, Nat. Neurosci. (2018). doi:10.1038/s41593018-0120-6.

[38] E.G. Hughes, J.L. Orthmann-Murphy, A.J. Langseth, D.E. Bergles, Myelin remodeling through experience-dependent oligodendrogenesis in the adult somatosensory cortex, Nat. Neurosci. (2018). doi:10.1038/s41593-018-0121-5.

[39] R.B. Tripathi, M. Jackiewicz, I.A. McKenzie, E. Kougioumtzidou, M. Grist, W.D. Richardson, Remarkable Stability of Myelinating Oligodendrocytes in Mice, Cell Rep. (2017). doi:10.1016/j.celrep.2017.09.050.

[40] K.M. Young, K. Psachoulia, R.B. Tripathi, S.J. Dunn, L. Cossell, D. Attwell, K. Tohyama, W.D. Richardson, Oligodendrocyte dynamics in the healthy adult CNS:

Evidence for myelin remodeling, Neuron. (2013). doi:10.1016/j.neuron.2013.01.006.

[41] E.M. Gibson, D. Purger, C.W. Mount, A.K. Goldstein, G.L. Lin, L.S. Wood, I. Inema, S.E. Miller, G. Bieri, J.B. Zuchero, B.A. Barres, P.J. Woo, H. Vogel, M. Monje, Neuronal activity promotes oligodendrogenesis and adaptive myelination in the mammalian brain, Science. (2014). doi:10.1126/science.1252304.

[42] S. Mitew, I. Gobius, L.R. Fenlon, S.J. McDougall, D. Hawkes, Y.L. Xing, H. Bujalka, A.L. Gundlach, L.J. Richards, T.J. Kilpatrick, T.D. Merson, B. Emery, Pharmacogenetic stimulation of neuronal activity increases myelination in an axon-specific manner, Nat. Commun. (2018). doi:10.1038/s41467-017-02719-2.

[43] J. Liu, K. Dietz, J.M. Deloyht, X. Pedre, D. Kelkar, J. Kaur, V. Vialou, M.K. Lobo, D.M. Dietz, E.J. Nestler, J. Dupree, P. Casaccia, Impaired adult myelination in the prefrontal cortex of socially isolated mice, Nat. Neurosci. (2012). doi:10.1038/nn.3263.

[44] M.M. Sánchez, E.F. Hearn, D. Do, J.K. Rilling, J.G. Herndon, Differential rearing affects corpus callosum size and cognitive function of rhesus monkeys, Brain Res. (1998). doi:10.1016/S0006-8993(98)00857-9. 
[45] M. Makinodan, K.M. Rosen, S. Ito, G. Corfas, A Critical Period for Social ExperienceDependent Oligodendrocyte Maturation and Myelination, Science. (2012). doi:10.1126/science. 1220845 .

[46] J.L. Sinclair, M.J. Fischl, O. Alexandrova, M. He $\beta$, B. Grothe, C. Leibold, C. KoppScheinpflug, Sound-Evoked Activity Influences Myelination of Brainstem Axons in the Trapezoid Body, J. Neurosci. (2017). doi:10.1523/JNEUROSCI.3728-16.2017.

[47] C. Sampaio-Baptista, A.A. Khrapitchev, S. Foxley, T. Schlagheck, J. Scholz, S. Jbabdi, G.C. DeLuca, K.L. Miller, A. Taylor, N. Thomas, J. Kleim, N.R. Sibson, D. Bannerman, H. Johansen-Berg, Motor Skill Learning Induces Changes in White Matter Microstructure and Myelination, J. Neurosci. (2013). doi:10.1523/JNEUROSCI.3048-13.2013.

[48] W. Feng, J. Wang, P.Y. Chhatbar, C. Doughty, D. Landsittel, V.A. Lioutas, S.A. Kautz, G. Schlaug, Corticospinal tract lesion load: An imaging biomarker for stroke motor outcomes, Ann. Neurol. (2015). doi:10.1002/ana.24510.

[49] B. Lakhani, K.S. Hayward, L.A. Boyd, Hemispheric asymmetry in myelin after stroke is related to motor impairment and function, NeuroImage Clin. (2017).

doi:10.1016/j.nicl.2017.01.009.

[50] M.R. Borich, A.L. MacKay, I.M. Vavasour, A. Rauscher, L.A. Boyd, Evaluation of white matter myelin water fraction in chronic stroke, NeuroImage Clin. (2013). doi:10.1016/j.nicl.2013.04.006.

[51] R. Dacosta-Aguayo, M. Graña, M. Fernández-Andújar, E. López-Cancio, C. Cáceres, N. Bargalló, M. Barrios, I. Clemente, P.T. Monserrat, M.A. Sas, A. Dávalos, T. Auer, M. Mataró, Structural integrity of the contralesional hemisphere predicts cognitive impairment in ischemic stroke at three months, PLoS One. (2014). doi:10.1371/journal.pone.0086119.

[52] C.E. Lang, J.M. Wagner, D.F. Edwards, A.W. Dromerick, Upper extremity use in people with hemiparesis in the first few weeks after stroke, J. Neurol. Phys. Ther. (2007). doi:10.1097/NPT.0b013e31806748bd.

[53] N. Langer, J. Hänggi, N.A. Müller, H.P. Simmen, L. Jäncke, Effects of limb immobilization on brain plasticity, Neurology. (2012). doi:10.1212/WNL.0b013e31823fcd9c.

[54] Y.-C. Lin, A. Daducci, D.E. Meskaldji, J.-P. Thiran, P. Michel, R. Meuli, G. Krueger, G. Menegaz, C. Granziera, Quantitative Analysis of Myelin and Axonal Remodeling in the Uninjured Motor Network After Stroke, Brain Connect. (2015). doi:10.1089/brain.2014.0245.

[55] F. Wang, Y.J. Yang, N. Yang, X.J. Chen, N.X. Huang, J. Zhang, Y. Wu, Z. Liu, X. Gao, T. Li, G.Q. Pan, S.B. Liu, H.L. Li, S.P.J. Fancy, L. Xiao, J.R. Chan, F. Mei, Enhancing Oligodendrocyte Myelination Rescues Synaptic Loss and Improves Functional Recovery after Chronic Hypoxia, Neuron. (2018). doi:10.1016/j.neuron.2018.07.017. 
[56] M. Nishibe, E.T.R. Urban, S. Barbay, R.J. Nudo, Rehabilitative training promotes rapid motor recovery but delayed motor map reorganization in a rat cortical ischemic infarct model, Neurorehabil. Neural Repair. (2015). doi:10.1177/1545968314543499.

[57] E.G. Sozmen, J.D. Hinman, S.T. Carmichael, Models That Matter: White Matter Stroke Models, Neurotherapeutics. (2012). doi:10.1007/s13311-012-0106-0.

[58] R.J.M. Franklin, C. Ffrench-Constant, Regenerating CNS myelin - From mechanisms to experimental medicines, Nat. Rev. Neurosci. (2017). doi:10.1038/nrn.2017.136.

[59] J. Smith, E. Ladi, M. Mayer-Proschel, M. Noble, Redox state is a central modulator of the balance between self-renewal and differentiation in a dividing glial precursor cell, Proc. Natl. Acad. Sci. (2000). doi:10.1073/pnas.170209797.

[60] C. Wang, Y. Zhang, J. Ding, Z. Zhao, C. Qian, Y. Luan, G.J. Teng, Nicotinamide Administration Improves Remyelination after Stroke, Neural Plast. (2017). doi:10.1155/2017/7019803.

[61] J. Ramos-Cejudo, M. Gutiérrez-Fernández, L. Otero-Ortega, B. Rodríguez-Frutos, B. Fuentes, M.T. Vallejo-Cremades, T.N. Hernanz, S. Cerdán, E. Díez-Tejedor, Brainderived neurotrophic factor administration mediated oligodendrocyte differentiation and myelin formation in subcortical ischemic stroke, Stroke. (2015). doi:10.1161/STROKEAHA.114.006692.

[62] E.G. Sozmen, S. Rosenzweig, I.L. Llorente, D.J. DiTullio, M. Machnicki, H. V. Vinters, L.A. Havton, R.J. Giger, J.D. Hinman, S.T. Carmichael, Nogo receptor blockade overcomes remyelination failure after white matter stroke and stimulates functional recovery in aged mice, Proc. Natl. Acad. Sci. (2016). doi:10.1073/pnas.1615322113.

[63] R.B. Tripathi, L.E. Rivers, K.M. Young, F. Jamen, W.D. Richardson, NG2 Glia Generate New Oligodendrocytes But Few Astrocytes in a Murine Experimental Autoimmune Encephalomyelitis Model of Demyelinating Disease, J. Neurosci. (2010). doi:10.1523/JNEUROSCI.3411-10.2010.

[64] D.E. Bergles, J.D.B. Roberts, P. Somogyl, C.E. Jahr, Glutamatergic synapses on oligodendrocyte precursor cells in the hippocampus, Nature. (2000). doi: $10.1038 / 35012083$.

[65] M. Kukley, E. Capetillo-Zarate, D. Dietrich, Vesicular glutamate release from axons in white matter, Nat. Neurosci. (2007). doi:10.1038/nn1850.

[66] J.L. Ziskin, A. Nishiyama, M. Rubio, M. Fukaya, D.E. Bergles, Vesicular release of glutamate from unmyelinated axons in white matter, Nat. Neurosci. (2007). doi:10.1038/nn1854.

[67] J. Müller, D. Reyes-Haro, T. Pivneva, C. Nolte, R. Schaette, J. Lübke, H. Kettenmann, The principal neurons of the medial nucleus of the trapezoid body and NG2 ${ }^{+}$glial cells receive coordinated excitatory synaptic input, J. Gen. Physiol. (2009). 
doi:10.1085/jgp.200910194.

[68] I. Lundgaard, A. Luzhynskaya, J.H. Stockley, Z. Wang, K.A. Evans, M. Swire, K. Volbracht, H.O.B. Gautier, R.J.M. Franklin, C. Ffrench-Constant, D. Attwell, R.T. Káradóttir, Neuregulin and BDNF Induce a Switch to NMDA Receptor-Dependent Myelination by Oligodendrocytes, PLoS Biol. (2013). doi:10.1371/journal.pbio.1001743.

[69] H.O.B. Gautier, K.A. Evans, K. Volbracht, R. James, S. Sitnikov, I. Lundgaard, F. James, C. Lao-Peregrin, R. Reynolds, R.J.M. Franklin, R.T. Káradóttir, Neuronal activity regulates remyelination via glutamate signalling to oligodendrocyte progenitors, Nat. Commun. (2015). doi:10.1038/ncomms9518.

[70] E. Dayan, L.G. Cohen, Neuroplasticity subserving motor skill learning, Neuron. (2011). doi:10.1016/j.neuron.2011.10.008.

[71] A. Etxeberria, J.M. Mangin, A. Aguirre, V. Gallo, Adult-born SVZ progenitors receive transient synapses during remyelination in corpus callosum, Nat. Neurosci. (2010). doi:10.1038/nn.2500.

[72] A. Sahel, F.C. Ortiz, C. Kerninon, P.P. Maldonado, M.C. Angulo, B. Nait-Oumesmar, Alteration of synaptic connectivity of oligodendrocyte precursor cells following demyelination, Front. Cell. Neurosci. (2015). doi:10.3389/fncel.2015.00077.

[73] G. Lin, A. Mela, E.M. Guilfoyle, J.E. Goldman, Neonatal and adult O4+oligodendrocyte lineage cells display different growth factor responses and different gene expression patterns, J. Neurosci. Res. (2009). doi:10.1002/jnr.22065.

[74] S. Marques, D. van Bruggen, D.P. Vanichkina, E.M. Floriddia, H. Munguba, L. Väremo, S. Giacomello, A.M. Falcão, M. Meijer, Å.K. Björklund, J. Hjerling-Leffler, R.J. Taft, G. Castelo-Branco, Transcriptional Convergence of Oligodendrocyte Lineage Progenitors during Development, Dev. Cell. (2018). doi:10.1016/j.devcel.2018.07.005.

[75] S.O. Spitzer, S. Sitnikov, Y. Kamen, K.A. Evans, D. Kronenberg-Versteeg, S. Dietmann, O. de Faria, S. Agathou, R.T. Káradóttir, Oligodendrocyte Progenitor Cells Become Regionally Diverse and Heterogeneous with Age, Neuron. (2019). doi:10.1016/J.NEURON.2018.12.020.

[76] D.H. Lentferink, J.M. Jongsma, I. Werkman, W. Baron, Grey matter OPCs are less mature and less sensitive to IFN $\gamma$ than white matter OPCs: Consequences for remyelination, Sci. Rep. (2018). doi:10.1038/s41598-018-19934-6.

[77] R. Chittajallu, A. Aguirre, V. Gallo, NG2-positive cells in the mouse white and grey matter display distinct physiological properties, J. Physiol. (2004). doi:10.1113/jphysiol.2004.074252.

[78] T. Kuhlmann, V. Miron, Q. Cuo, C. Wegner, J. Antel, W. Brück, Differentiation block of oligodendroglial progenitor cells as a cause for remyelination failure in chronic multiple sclerosis, Brain. (2008). doi:10.1093/brain/awn096. 
[79] J.M. Ruckh, J.W. Zhao, J.L. Shadrach, P. Van Wijngaarden, T.N. Rao, A.J. Wagers, R.J.M. Franklin, Rejuvenation of regeneration in the aging central nervous system, Cell Stem Cell. (2012). doi:10.1016/j.stem.2011.11.019.

[80] G. Wolswijk, W.-W. Li, C. Zhao, R.J.M. Franklin, Chronic stage multiple sclerosis lesions contain a relatively quiescent population of oligodendrocyte precursor cells., J. Neurosci. (1998). doi:10.1016/S1071-9091(98)80030-2. 


\section{Figure Legend}

Figure 1: Oligodendrocyte precursor cell states might play a central role in remyelination and myelin plasticity after stroke.

(A) Oligodendrocyte precursor cells (OPCs) first appear as 'naïve' cells (light grey) without ion channels. They transition through an intermediate state (pink), acquiring voltage-gated potassium channels $\left(\mathrm{K}_{\mathrm{V}}\right)$ and AMPA/kainate receptors (AMPA/KARs), before becoming 'highly proliferative' (yellow), when they express high densities of voltage-gated sodium ( $\mathrm{Na}_{\mathrm{V}}$ ) channels. Later, OPCs express high densities of NMDA receptors (NMDARs) and become 'primed' for differentiation (blue). During ageing, OPCs become 'quiescent' (dark grey), with high AMPA/KAR densities, but have lost NMDARs and their ability to differentiate.

(B) Demyelination and remyelination in the penumbra after stroke. In young adults, most OPC states are present in the penumbra, so OPCs can proliferate and differentiate into myelinating oligodendrocytes (OLs) to replace those damaged by injury. With age, the majority of OPCs become quiescent and lose their ability to differentiate, leading to potential remyelination failure.

(C) Axonal rewiring and de novo myelination in the penumbra and peri-infarct after stroke. After $\mathrm{OL}$ and neuronal death, surviving neurons rewire and retarget to replace lost synapses (yellow branches). New myelinating OLs (light blue) are generated to strengthen new neuronal circuits. In old mice, the absence of highly proliferative and primed OPCs and the large number of quiescent OPCs presumably prevent differentiation and de novo myelination. 


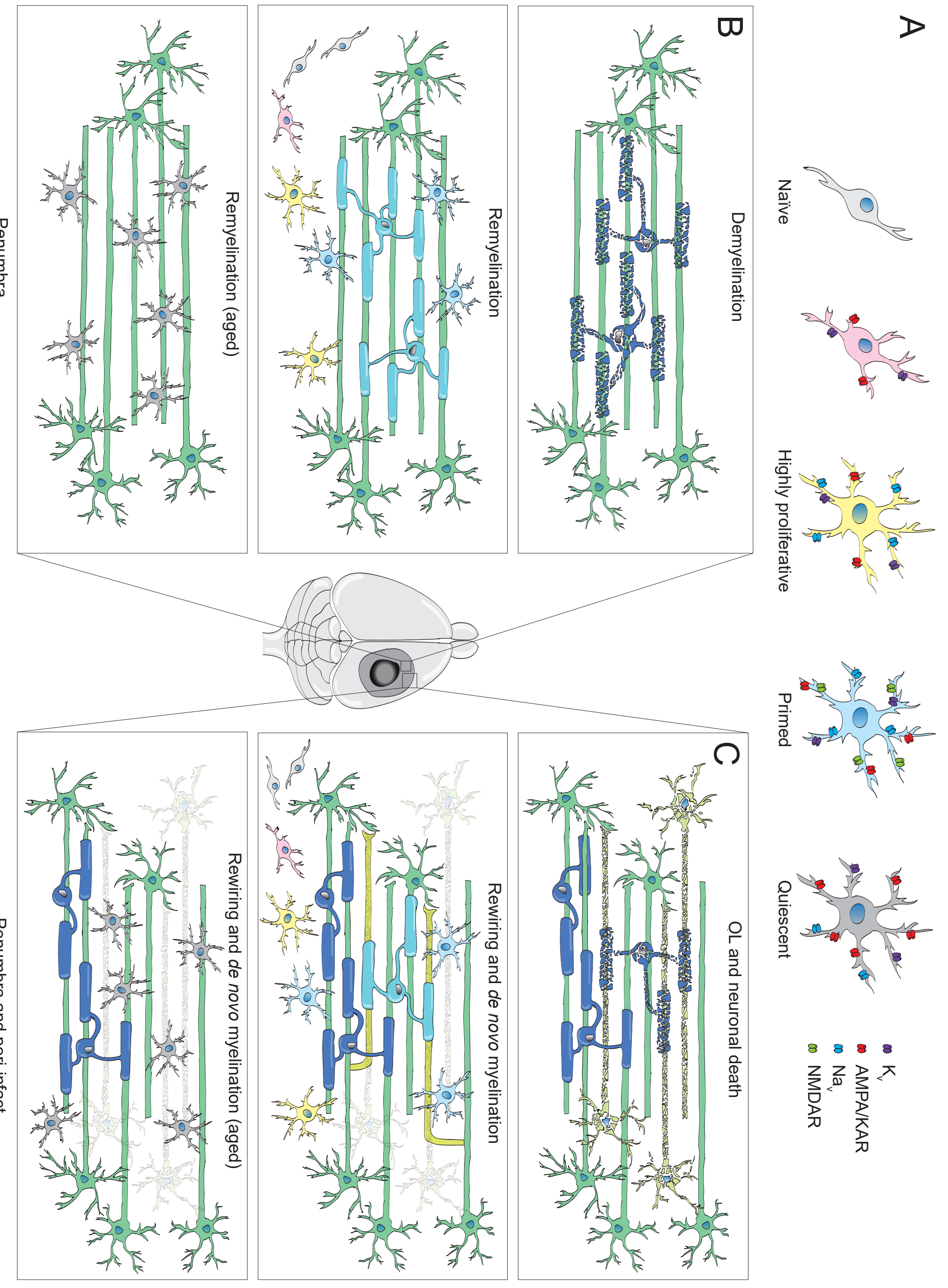

